\title{
X-ray Phase Contrast Microscopy Based on Parabolic Refractive Axicon Lens.
}

\author{
D. Zverev ${ }^{1}, \underline{\text { I. Snigireva }}{ }^{2, *}$ and A. Snigirev ${ }^{1}$ \\ 1. Immanuel Kant Baltic Federal University, Kaliningrad, Russia \\ 2. European Synchrotron Radiation Facility, Grenoble, France \\ *irina@esrf.fr
}

The most advanced X-ray sources, such as third-generation synchrotrons and free electron lasers, are capable to generate high brightness coherent radiation, especially in the hard X-ray region. The availability of such beams facilitates to the development of a new generation of X-ray optics, which goes far beyond simple focusing optical elements. This optics possess a new optical functions which allowed to form the intensity of the wave front with almost complete freedom. Such beam-shaping capabilities utilize the most outstanding properties of synchrotron radiation such as brightness, monochromaticity and coherence.

Recently, the X-ray parabolic refractive axicon was demonstrated as a novel type of X-ray beam-shaping optical element [1]. The single parabolic axicon represents a biconcave refractive lens with two conical parabolic cavities. Under coherent X-ray illumination, the axicon generates Bessel-like beam along the optical axis in the near and ring-shaped beam in the far fields. The shaped beams produced by the axicon can be used in areas requiring extended focused beams, as well as special illumination, for instance, in diffraction and imaging techniques. The use of the axicon lenses can significantly simplify some experimental layouts or lead to completely new optical schemes for new X-ray techniques based on the synchrotron. In this paper, we present a new approach to the phase contrast imaging technique, which takes advantages of the traditional X-ray microscopy and the unique optical properties of the parabolic refractive axicon.

Today, X-ray microscopy based on compound refractive lenses (CRLs) is one of the most attractive technique for obtaining high-resolution imaging at high energy range. It is used for nondestructive visualization the features of the internal structure of the objects in material science, biology, and medicine. However, the main contrast formation is absorption that creates some limitations for imaging of samples consisting of light-density materials. Such samples are almost transparent for hard X-rays, and they are actually phase objects. Therefore, the use of a phase contrast mechanism for image formation becomes a necessity.

The contrast of the image of the phase object can be increased by using special optical schemes that allow the phase modulations caused by the sample to be converted in modulations of the intensity in the image plane. It is well known in optics that the phase contrast was realized in the microscopy proposed by Zernike. In this case the phase-contrast image is formed by interference between the reference wave front, which is phase-shifted by a phase plate, and a wave that diffract on the sample. This approach has already been applied for microscopy in the hard X-ray region allowing to significantly expand the capabilities of traditional X-ray microscopy technique [2-3].

Usually, the configuration of Zernike X-ray phase contrast microscopy experimental setup is rather complicated. For example, to separate the interfering beams, a special ring-type illumination of the sample obtained using a beam stop device or an annular aperture is applied, and therefore a time-consuming adjustment and alignment of the optical scheme is required. In addition, the design of the phase plate is 
calculated only for a specific wavelength because the phase shift of the reference wave should be equal to half of $\pi$. Moreover, in some cases there are additional difficulties associated with the fabrication limitations. The use of a parabolic axicon lens as a phase shifted device allows to fully overcome these shortcomings. In this case the parabolic axicon provides a smooth phase shift of the wave diffracted by the phase sample. The axicon can operate over a wide range of energies, while sophisticated annular illumination is not required.

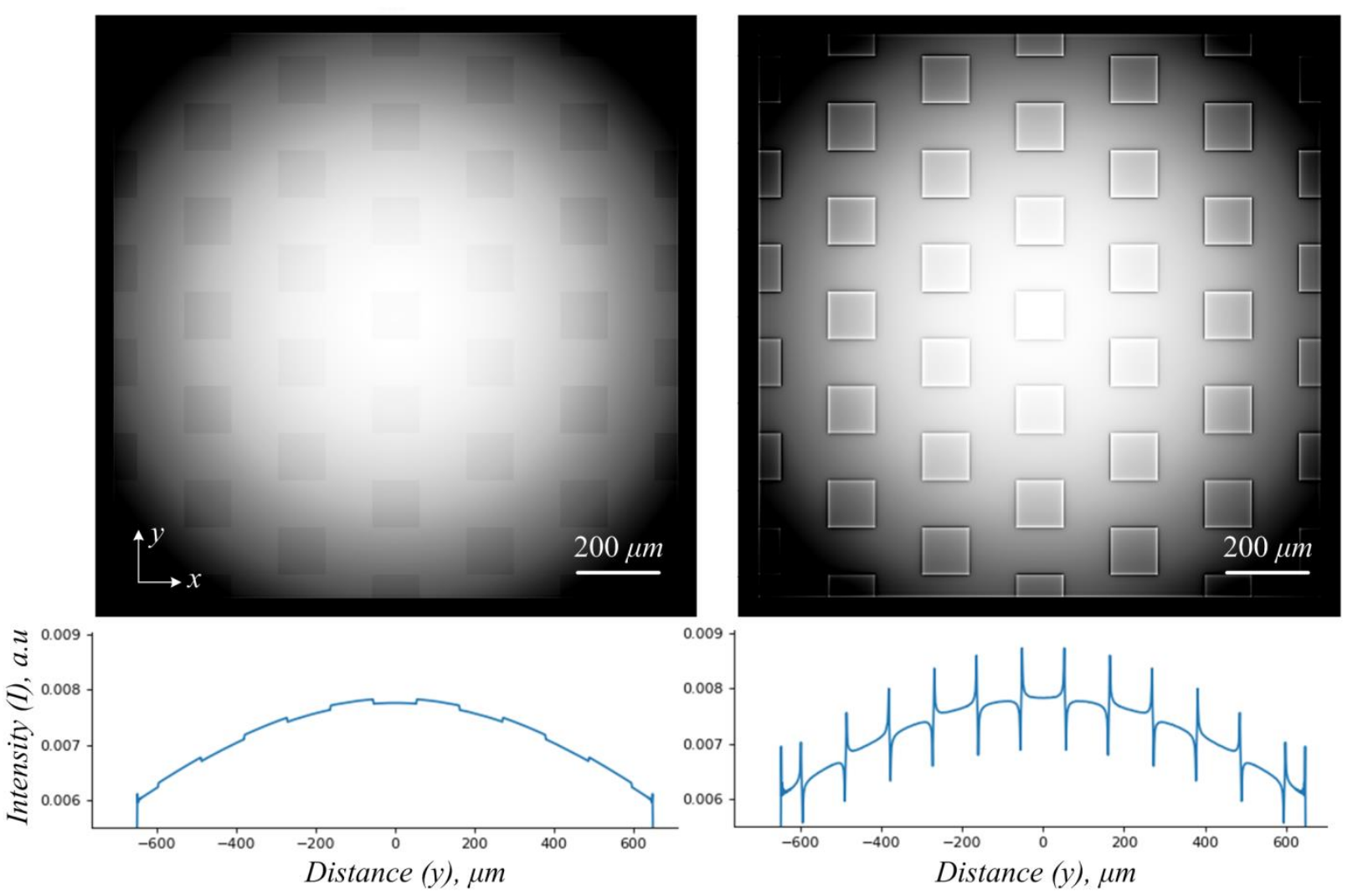

Figure. 1. Computer simulated images of the phase grating in the schemes: (left) traditional X-ray microscopy technique and (right) X-ray phase contrast microscopy based on the parabolic axicon lens

A computer modelling of the proposed approach of phase-contrast X-ray microscopy was carried out. Numerically calculated X-ray images of a phase grating obtained using X-ray microscopy in traditional and phase contrast schemes are presented in Fig. 1. It is evident that X-ray phase contrast microscopy, based on the parabolic axicon lens, is more effective for visualizing weakly absorbing samples compared to the traditional microscopy technique [4].

\section{References:}

[1] D. Zverev, A. Barannikov, I. Snigireva, A. Snigirev, Optics Express 25 (2017) 28469-28477.

[2] C.Holzner et al, Nature Physics 6 (2010) 883-887.

[3] K. Falch et al, Ultramicroscopy 184 (2018) 267-273.

[4] The authors acknowledge funding from the Ministry of Education and Science of the Russian Federation grant contract № 14.Y26.31.0002. 